\title{
Performance of a HRP-2/pLDH based rapid diagnostic test at the Bangladesh-India-Myanmar border areas for diagnosis of clinical malaria
}

\author{
Rubayet Elahi ${ }^{1,2}$, Abu Naser Mohon ${ }^{1}$, Wasif A Khan ${ }^{1}$, Rashidul Haque ${ }^{1}$ and Mohammad Shafiul Alam ${ }^{\text {** }}$
}

\begin{abstract}
Background: The rapid diagnostic test (RDT) has been adopted in contemporary malaria control and management programmes around the world as it represents a fast and apt alternative for malaria diagnosis in a resource-limited setting. This study assessed the performance of a HRP-2/pLDH based RDT (Parascreen ${ }^{\circledR}$ Pan/Pf) in a laboratory setting utilizing clinical samples obtained from the field.
\end{abstract}

Methods: Whole blood samples were obtained from febrile patients referred for malaria diagnosis by clinicians from two different Upazila Health Complexes (UHCs) located near the Bangladesh-India and Bangladesh-Myanmar border where malaria is endemic. RDT was performed on archived samples and sensitivity and specificity evaluated with expert microscopy (EM) and quantitative PCR (qPCR).

Results: A total of 327 clinical samples were made available for the study, of which 153 were Plasmodium falciparum-positive and 54 were Plasmodium vivax-positive. In comparison with EM, for $P$. falciparum malaria, the RDT had sensitivity: $96.0 \%$ (95\% Cl, 91.2-98.3) and specificity: $98.2 \%$ (95\% Cl, 94.6-99.5) and for P. vivax, sensitivity: $90.7 \%$ ( $95 \%$ Cl, 78.9-96.5) and specificity: 98.9\% (95\% Cl, 96.5-99.7). Comparison with qPCR showed, for P. falciparum malaria, sensitivity: $95.4 \%$ (95\% Cl, 90.5-98.0) and specificity: $98.8 \%$ (95\% Cl, 95.4-99.7) and for P. vivax malaria, sensitivity: 89.0\% (95\% Cl,77.0-95.4) and specificity: 98.8\% (95\% Cl, 96.5-99.7). Sensitivity varied according to different parasitaemia for falciparum and vivax malaria diagnosis.

Conclusion: Parascreen ${ }^{\circledR}$ Pan/Pf Rapid test for malaria showed acceptable sensitivity and specificity in border belt endemic areas of Bangladesh when compared with EM and GPCR.

\section{Background}

Malaria is often lethal with high potential expenditure for health if diagnosis is inaccurate [1]. Accurate diagnosis of malaria is of increasing importance as the prevalence of malaria is declining around the globe, making surveillance and screening more important for programme management $[2,3]$ and to restrict the use of anti-malarial drugs to restrain the spread of drug resistance [4].

For decades, expert microscopy (EM) of peripheral thick and thin blood smears has been the standard diagnostic test for malaria, however, it is time consuming and requires substantial expertise [1,5]. Enzyme-linked immunosorbent assay (ELISA) and polymerase chain reaction (PCR)-based

\footnotetext{
* Correspondence: shafiul@icddrb.org

'International Centre for Diarrhoeal Disease Research Bangladesh (icddr,b), Dhaka, Bangladesh

Full list of author information is available at the end of the article
}

diagnostic tests have been introduced which ameliorate sensitivity and specificity of malaria diagnosis, but only in reference settings where well equipped laboratory facilities are available, making it difficult to implement in a field setting [6]. Other nascent molecular methods, such as loop-mediated isothermal amplification (LAMP) [7-9] and real-time quantitative nucleic acid sequence-based amplification (QT-NASBA) [10] are in use, but the efficacy of each is unproven.

After being introduced in the early 1990s, rapid diagnostic tests (RDTs) have become an attractive alternative to the above-mentioned methods in a resource-limited setting for malaria diagnostics. The antigen-based RDTs detect specific antigens produced by malaria parasites by reaction with bound antibodies on an absorbent nitrocellulose membrane. Among several types of RDTs the two-band tests and three-band tests are most 
widely used. Two-band tests either detect only one species (Plasmodium falciparum), usually by detecting histidine-rich protein 2 (HRP2), or detect any of the four most common malaria parasites (P. falciparum, Plasmodium vivax, Plasmodium malariae and Plasmodium ovale), typically by detecting pan-Plasmodium-specific lactate dehydrogenase (pLDH), while three-band tests detect both the P. falciparum-specific antigen HRP2 and the pLDH or any one species specific LDH (mostly $P$. vivax). The third band is the test control band $[1,5,11]$.

Southeast Bangladesh, northeast India and southwest Myanmar are similar in geographical characteristics and endemic for malaria. Plasmodium falciparum is the most abundant parasite, followed by $P$. vivax in these countries $[6,11,12]$. The presence of $P$. malariae and $P$. ovale has also been reported in each country [13-16]. These three countries share their borders, making transborder malaria transmission plausible. The presence of all four parasites in these mostly remote and resource-limited areas illustrate the importance of a RDT that can detect all malaria parasites. Amongst the locally available RDTs, Parascreen ${ }^{\circledR}$ Pan/Pf Rapid test for malaria (Zephyr Biomedical Systems, India), hereafter noted as Parascreen, is a RDT that has the capability to detect all types of human malaria, as it detects P. falciparum-specific HRP-2 and pan-Plasmodium-specific LDH. It has been evaluated against microscopy and conventional PCR in field and laboratory settings [12,17-24]. Here, the assessment of Parascreen in a laboratory setting and its performance compared with EM and qPCR are described.

\section{Methods}

\section{Study area and population}

Whole blood samples were obtained from febrile patients with clinical symptoms referred for laboratory investigation between May 2009 to December 2010. The represented regions include Matiranga Upazila in Khagrachari district and Ramu Upazila in Cox's Bazar district, two different subdistricts of the southeastern part of Bangladesh from corresponding UHC. Matiranga borders Tripura state of India and Ramu borders Myanmar, where malaria is endemic $[15,16]$ and is caused mainly by $P$. falciparum and $P$. vivax.

\section{Sample collection}

An expert medical technologist collected approximately $5 \mathrm{~mL}$ of blood from adult subjects and $3 \mathrm{~mL}$ from minor subjects by venipuncture. Thick and thin blood films were prepared in duplicate using two drops of blood for each sample. The remaining blood was preserved at $-20^{\circ} \mathrm{C}$ in EDTA tube and transported to the Parasitology Laboratory, icddr,b in cool boxes maintaining the temperature below $4^{\circ} \mathrm{C}$ using ice bags.
Approval from Research Review Committee (RRC) and Ethical Review Committee (ERC) of icddr,b was obtained for this study. Permission for conducting the study was obtained from the National Malaria Control Programme (NMCP). All participants or legal guardians signed informed consent before participant enrolment and sample collection. Complete anonymity was maintained at each stage of the study.

\section{Expert microscopy (EM)}

Blood smears were stained with Giemsa and screened for parasites under the (100X) oil immersion lens at the field site by experienced microscopists in the corresponding UHC. The microscopy results were confirmed by a second independent, experienced microscopist who was blinded to prior results. Parasite density was determined by both microscopists counting the parasites and leucocytes [25] and the average was used for the study. When there was any disagreement in diagnosis by the two microscopists for any sample and mixed ( $P$. falciparum and $P$. vivax) infection were excluded from the study.

\section{Rapid diagnostic tests (RDTs)}

Parascreen (Zephyr Biomedical Systems, India; Lot No 101159) is a three-band antigen detection RDT which comes in cassette format. It employs a recombinant antibody against pLDH to detect Plasmodium-specific LDH and anti-HRP2 antibody to detect $P$. falciparumspecific HRP2. All RDTs were performed on archived blood samples by trained and skilled laboratory personnel at the Parasitology Laboratory, icddr,b following the manufacturer's instructions. Briefly, one pink-purple line in the proximal area (control line) interprets negative for malaria; one pink-purple line in the middle area, along with the control line, interprets non-P. falciparum infection, exclusively $P$. vivax in this study; one pink-purple line, along with the previous two bands, interprets $P$. falciparum infection. If any of the two test lines or control line did not appear, the test was invalid and repeated.

\section{DNA isolation}

DNA was isolated using QIAamp DNA blood mini kit (Qiagen Sciences Inc, USA) following the manufacturer's instructions from $200 \mu \mathrm{L}$ of archived whole blood.

qPCR

Quantitative PCR (qPCR) was performed on isolated DNA following the method described by Alam et al. [6] with Invitrogen ${ }^{\circledR}$ SYBR Green I supermix UDG (Life Technologies Corporation, USA). The sensitivity and specificity of qPCR for P. falciparum was 97.1 and $97.6 \%$, respectively, while for $P$. vivax 95.2 and $98.1 \%$ [6]. Any mixed ( $P$. falciparum and $P$. vivax) infection diagnosed by qPCR was not considered in this study. 


\section{Data analysis}

All data were encoded in an Excel data sheet and the performance of RDT was calculated by means of the following indicators: sensitivity, specificity, positive predictive value (PPV), negative predictive value (NPV) and agreement (kappa) were calculated with their corresponding 95\% confidence intervals (95\% CI), using EM and qPCR as reference standards. Sensitivity was calculated as the proportion of positive RDT test results among malaria-positive samples identified by EM and qPCR, while specificity was calculated as the proportion of negative test results among the malaria-negative samples identified by the reference standards. PPV and NPV were obtained as the true positive results among all malaria-positive samples and the true negative results among all negative samples, respectively [26]. Agreement (k) analysis was conducted in IBM SPSS Statistics, version 17.0 (IBM Corporation, NY, USA) by creating a $2 \times 2$ contingency table.

\section{Results}

In total, 327 febrile patients were included in this study from two UHCs. The results of EM, qPCR and Parascreen are provided in Table 1. With EM, there were 207 (63.3\%) positive malaria cases, of which 153 (73.9\%) were $P$. falciparum infection and $54(26.0 \%)$ were $P$. vivax infection. The parasite density for $P$. falciparum ranged between 16 and 261,480 parasites/ $\mu \mathrm{L}$ (IQR: $7,500-50,100)$ with median value of 19,960 parasites/ $\mu \mathrm{L}$, while the parasite density for $P$. vivax ranged between 16 and 25,120 parasites/ $\mu \mathrm{L}$ (IQR: 320-4,800) with median value of 1,140 parasites/ $\mu \mathrm{L}$. qPCR confirmed 208 (63.6\%) positive malaria cases, of which 154 (74.0\%) were $P$. falciparum and 54 (25.9\%) were $P$. vivax. With Parascreen, there were $202(61.7 \%)$ malaria positive cases, of which 150 (74.2\%) were P. falciparum and 52 (25.7\%) were $P$. vivax infection.

Table 2 represents the calculated indicators when Parascreen was compared with EM and qPCR. EM being the reference standard, Parascreen had the following results, for any kind of malaria detection, sensitivity: $97.1 \%$ (95\% CI, 93.5-98.8) and specificity: 99.1\% (95\% CI, 96.899.9); for $P$. falciparum malaria detection, sensitivity: 96.0\% (95\% CI, 91.2-98.3) and specificity: 98.2\% (95\% CI,

Table 1 Parascreen ${ }^{\circledR}$ diagnosis results and comparison with diagnosis by EM and qPCR

\begin{tabular}{|c|c|c|c|c|c|c|c|}
\hline \multirow{2}{*}{$\begin{array}{l}\text { Parasreen } \\
\text { results }\end{array}$} & & \multicolumn{3}{|c|}{ Microscopy } & \multicolumn{3}{|l|}{ qPCR } \\
\hline & & Negative & $\mathrm{Pf}$ & $\mathrm{Pv}$ & Negative & Pf & Pv \\
\hline Negative & 125 & 119 & 3 & 3 & 119 & 3 & 3 \\
\hline$P f$ & 150 & 1 & 147 & 2 & 0 & 148 & 2 \\
\hline PV & 52 & 0 & 3 & 49 & 0 & 3 & 49 \\
\hline Total & 327 & 120 & 153 & 54 & 119 & 154 & 54 \\
\hline
\end{tabular}

94.6-99.5) and for P. vivax malaria detection, sensitivity: 90.7\% (95\% CI, 78.9-96.5) and specificity: 98.9\% (95\% CI, 96.5-99.7). When qPCR was used as the reference standard, Parascreen had the following results for any kind of malaria detection, sensitivity: 97.1\% (95\% CI, 93.5-98.8) and specificity: 100\% (96.1-100.0); for P. falciparum malaria detection, sensitivity: $95.4 \%$ (95\% CI, 90.5-98.0) and specificity: $98.8 \%$ (95\% CI, 95.4-99.7) and for P. vivax malaria detection, sensitivity: $89.0 \%$ (95\% CI, 77.0-95.4) and specificity: 98.8\% (95\% CI, 96.5-99.7).

Parascreen showed higher sensitivity (93.3-100\%) in detecting samples with parasite densities $>500$ parasites/ $\mu \mathrm{L}$ for both $P$. falciparum and $P$. vivax, whereas for parasite densities ranging from 1-500 parasites/ $\mu \mathrm{L}$, the sensitivity was low (60.0\%-83.3\%) (Figure 1 ).

\section{Discussion}

Parascreen showed acceptable performance in this study with overall sensitivity and specificity of 97.1 and $99.1 \%$, respectively, when compared to EM, and 97.1 and 100\%, respectively, in comparison with qPCR. Parascreen can detect all types of non-falciparum malaria but in this study only $P$. vivax was considered as $P$. malariae and $P$. ovale cases were not present in the study samples $[13,14]$. Parascreen demonstrated varying sensitivity and specificity when compared with EM and qPCR depending on parasite species (P. falciparum and $P$. vivax) and parasitaemia of infections.

Several evaluation studies of Parascreen in different countries reported overall sensitivity ranging from 47.5 to $95.5 \%$ and specificity from 64.3 to $98.5 \%$ with varying performance for falciparum and non-falciparum malaria detection [12,17-21,23,24]. Parascreen has been evaluated by WHO RDT evaluation programme and for $P$. falciparum detection it showed almost $100 \%$ detection rates while for $P$. vivax it was approximately $30 \%$ [27]. Here, in this study, for $P$. falciparum detection, the sensitivity and specificity was also in concordance with the previous findings [12,20,21], while for P. vivax detection, improved sensitivity and specificity are reported. The improved sensitivity and specificity of $P$. vivax detection compared to previous findings may be due to the increased release of antigen through parasite lysis in the archived sample [1] or due to the improvement in the product. This finding is also corroborated by a meta analysis where mean sensitivity and specificity of 95.0 and 95.2\%, respectively, for HRP-2 based assays and 93.2 and $98.5 \%$, respectively, for $\mathrm{pLDH}$ based assays were calculated [28].

In a study in India, Parascreen showed 94.0\% sensitivity and $72.0 \%$ specificity for $P$. falciparum and for $P$. vivax $77.2 \%$ sensitivity and $98.1 \%$ specificity were recorded when compared with EM and similar values observed when compared with PCR [12]. 
Table 2 Comparative indicators of Parascreen ${ }^{\circledR}$, when using EM and qPCR as reference standard

\begin{tabular}{lllllll}
\hline $\begin{array}{l}\text { Reference } \\
\text { standard }\end{array}$ & Test & \multicolumn{5}{c}{ Results by Parascreen } \\
\cline { 3 - 7 } & & Sensitivity [\%(95\% CI)] & Specificity [\%(95\% CI)] & PPV [\%(95\% Cl)] & NPV [\%(95\% Cl)] & Agreement (k) \\
\hline \multirow{2}{*}{ EM } & Overall & $97.1(93.5-98.8)$ & $99.1(94.7-99.9)$ & $99.5(96.8-99.9)$ & $95.2(89.4-98.0)$ & 0.954 \\
& Pf & $96.0(91.2-98.3)$ & $98.2(94.6-99.5)$ & $98.0(93.8-99.4)$ & $96.6(92.4-98.6)$ & 0.945 \\
& Pv & $90.7(78.9-96.5)$ & $98.9(96.5-99.7)$ & $94.2(83.0-98.4)$ & $98.1(95.5-99.3)$ & 0.910 \\
& Overall & $97.1(93.5-98.8)$ & $100.0(96.1-100.0)$ & $100.0(97.6-100.0)$ & $95.2(89.4-98.0)$ & 0.961 \\
\multirow{2}{*}{ qPCR } & Pf & $95.4(90.5-98.0)$ & $98.8(95.4-99.7)$ & $98.6(94.7-99.7)$ & $96.0(94.7-99.7)$ & 0.945 \\
& Pv & $89.0(77.0-95.4)$ & $98.8(96.5-99.7)$ & $94.2(83.0-98.4)$ & $97.8(95.0-99.1)$ & 0.899 \\
\hline
\end{tabular}

In Myanmar, two RDTs with similar detection properties have been evaluated in field settings [11,26]. The SD 05FK60 RDT evaluated in the Rakhaine state of Myanmar showed $90.2 \%$ sensitivity and $98.5 \%$ specificity for P. falciparum and $79.4 \%$ sensitivity and $98.7 \%$ specificity for non-falciparum malaria [11]. The VIKIA Malaria Ag Pf/ $\operatorname{Pan}^{\text {TM }}$ test showed 98.0 and 100\% sensitivity for $P$. falciparum and non-falciparum malaria, respectively, with specificity of 98.0 and $100 \%$, respectively [26].

Onsite (Pf/Pan), a RDT with similar detection principle recently evaluated in Bangladesh, reported 94.2\% sensitivity and $99.5 \%$ specificity for falciparum malaria detection and for vivax malaria detection it showed sensitivity and specificity of 97.3 and $98.7 \%$, respectively [5] which showed slightly better sensitivity and specificity compared to Parascreen.
WHO recommends sensitivity $\geq 95 \%$ at $\geq 100$ parasites/uL for RDTs [1]. In this study, for both falciparum and vivax malaria detection, sensitivity was less than the recommended values for low parasitaemia; however, considering fewer low parasitaemia samples, statistically valid conclusions have not been attained.

In this study, Parascreen was unable to detect three microscopically confirmed falciparum malaria samples with parasitaemia ranging from 112 to 2,600 parasites/ uL. This might be caused by the degradation of HRP-2 target antigen as the study was carried out with archived samples. Intraspecies sequence variation [29], deletions or mutations of HRP-2 gene [30,31] among different $P$. falciparum isolates could also account for false negative tests. The extent of HRP-2 variations in Bangladesh is currently unknown, however variations or deletions in

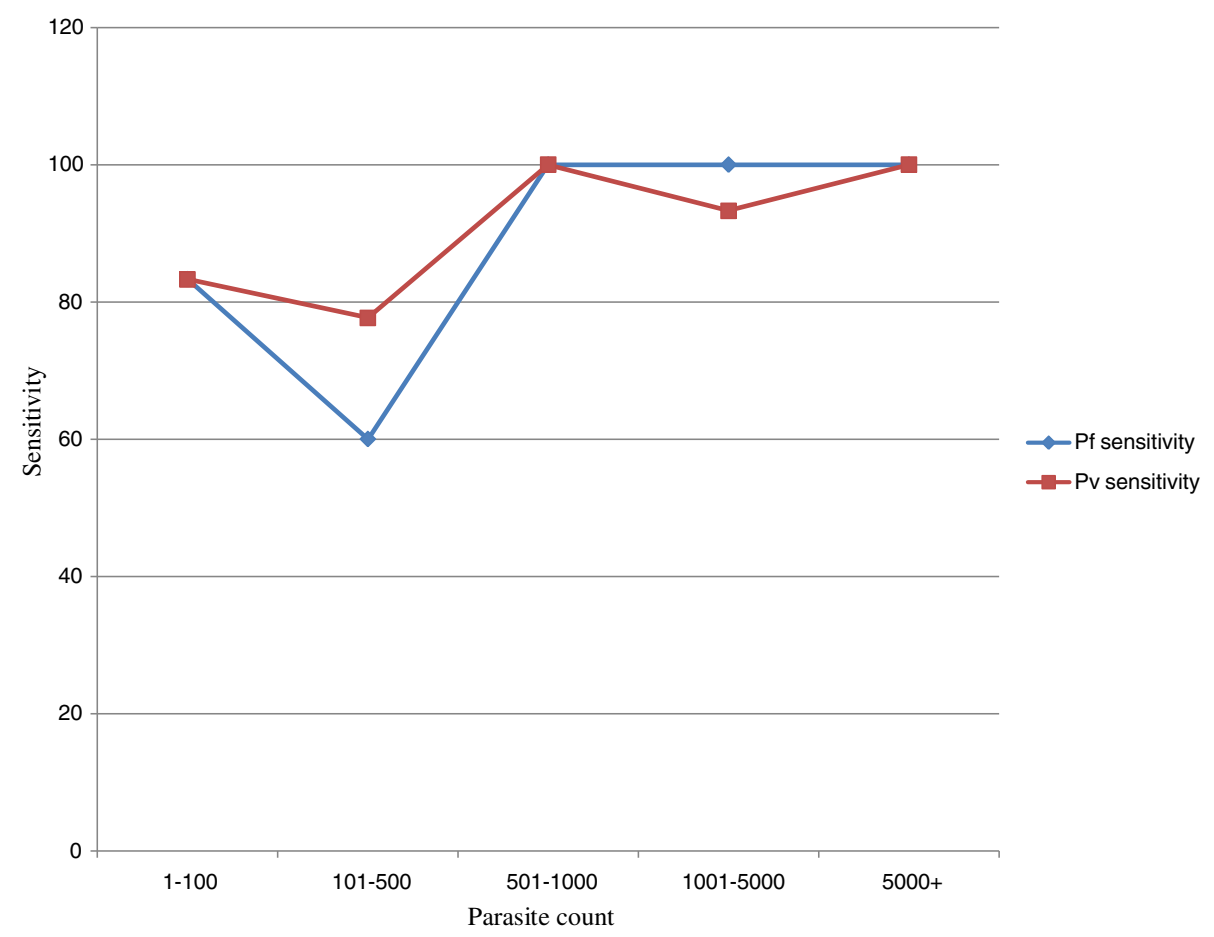

Figure 1 Varied sensitivity of Parascreen ${ }^{\circledR}$ (Pf/pan) according to different parasitaemia. 
HRP-2 have been reported recently from India, [31] as well as some African countries [30,32]. In this study, three $P$. falciparum samples showed no HRP-2 test line but Pan specific test line, thus considered as $P$. vivax, as other types of malaria were absent in the study samples. The intraspecies variation, mutation or deletions in the HRP-2 gene can cause non-expression of HRP-2 [30] which may explain this. Parascreen identified three $P$. vivax-positive samples with parasitaemia ranging from 16 to 200 parasites/uL as negative. This might be due to low pLDH level, as pLDH level is directly proportional to parasitaemia [33]. In many studies, a reduced sensitivity for non-falciparum malaria detection, compared to falciparum detection, in combined HRP-2/pLDH RDTs has also been reported $[5,6,11,26]$.

As all four malarial parasites co-exist in the BangladeshIndia-Myanmar border area, an important criterion for selection of an appropriate RDT is the capability to detect all types of malaria. It is advantageous to use Pf/Pan RDTs which can do so. The high predictive values for Parascreen indicate that it is able to detect true malaria cases as well as ruling out non-malaria cases. High sensitivity, specificity and predictive values for Parascreen present it as a viable alternative for malaria diagnostics in Bangladesh-India-Myanmar border areas where malaria is endemic.

The absence of $P$. malariae and $P$. ovale samples in this study restricts the findings to the detection performance of falcipaum and vivax malaria. The inclusion of $P$. malariae and $P$. ovale in the study samples is needed to assess non-falciparum malaria detection performance.

\section{Conclusion}

Parascreen showed acceptable performance for falciparum as well as vivax malaria diagnosis in standard experimental conditions. It can be employed in resource-limited settings to diagnose all types of human malaria.

\section{Competing interests}

The authors declare that they have no competing interests.

\section{Authors' contributions \\ RE and MSA conceptualized and designed the study, collected and identified samples, analyzed the data, drafted the manuscript and made final revisions. RE, ANM and MSA did sample analysis. WAK and RH made critical revision of the manuscript. RE and ANM performed laboratory tests. All the authors read the final version of the manuscript and approved.}

\section{Acknowledgements}

This research study was funded by icddr,b and its donors, which provide unrestricted support to icddr,b for its operations and research. Current donors providing unrestricted support include: Australian Agency for International Development (AusAID), Government of the People's Republic of Bangladesh; Canadian International Development Agency (CIDA), Swedish International Development Cooperation Agency (Sida), and the Department for International Development, UK (DFID). We gratefully acknowledge these donors for their support and commitment to icddr,b's research efforts. We are thankful to Zephyr Biomedical Systems, India for providing Parascreen ${ }^{\circledR}$ as a donation.
We are grateful to the NMCP for their permission to conduct the study in their facilities. We are indebted to the people of Matiranga and Ramu who consented to participate in the study and the doctors and staff of Matiranga and Ramu UHC for their extended support. We acknowledge Kevin E

McElhanon for kindly editing the manuscript. We appreciate the contribution of Shariar Mustafa, Khaja Md Mohiuddin, Mamun Kabir, H M Al-Amin and Milka Patracia Podder for their valuable contributions to the study.

\section{Author details}

${ }^{1}$ International Centre for Diarrhoeal Disease Research Bangladesh (icddr,b), Dhaka, Bangladesh. ${ }^{2}$ Department of Neurobiology and Developmental Sciences, University of Arkansas for Medical Sciences (UAMS), Little Rock, AR, USA.

Received: 2 September 2013 Accepted: 28 October 2013

Published: 30 October 2013

\section{References}

1. Bell D, Peeling RW: WHO-regional office for the WesternPacific/TDR: evaluation of rapid diagnostic tests: malaria. Nat Rev Microbiol 2006, 4:S34-S40.

2. Feachem RG, Phillips AA, Hwang J, Cotter C, Wielgosz B, Greenwood BM, Sabot O, Rodriguez MH, Abeyasinghe RR, Ghebreyesus TA, Snow RW: Shrinking the malaria map: progress and prospects. Lancet 2010, 376:1566-1578.

3. Moonen B, Cohen JM, Snow RW, Slutsker L, Drakeley C, Smith DL, Abeyasinghe RR, Rodriguez MH, Maharaj R, Tanner M, Targett G: Operational strategies to achieve and maintain malaria elimination. Lancet 2010, 376:1592-1603.

4. Murray CK, Bennett JW: Rapid diagnosis of malaria. Interdiscip Perspect Infect Dis 2009, 2009:415953.

5. Mohon AN, Elahi R, Podder MP, Mohiuddin K, Hossain MS, Khan WA, Haque R, Alam MS: Evaluation of the OnSite (Pf/Pan) rapid diagnostic test for diagnosis of clinical malaria. Malar J 2012, 11:415

6. Alam MS, Mohon AN, Mustafa S, Khan WA, Islam N, Karim MJ, Khanum H, Sullivan DJ, Haque R: Real-time PCR assay and rapid diagnostic tests for the diagnosis of clinically suspected malaria patients in Bangladesh. Malar J 2011, 10:175.

7. Hopkins H, González IJ, Polley SD, Angutoko P, Ategeka J, Asiimwe C, Agaba B, Kyabayinze DJ, Sutherland CJ, Perkins MD, Bell D: Highly sensitive detection of malaria parasitemia in a malaria-endemic setting: performance of a new loop-mediated isothermal amplification kit in a remote clinic in Uganda. J Infect Dis 2013, 208:645-652.

8. Abdul-Ghani R, Al-Mekhlafi AM, Karanis P: Loop-mediated isothermal amplification (LAMP) for malarial parasites of humans: would it come to clinical reality as a point-of-care test? Acta Trop 2012, 122:233-240.

9. Tao Z-Y, Zhou H-Y, Xia H, Xu S, Zhu H-W, Culleton RL, Han E-T, Lu F, Fang Q, Gu Y-P, Liu Y-B, Zhu G-D, Wang W-M, Li J-L, Cao J, Gao Q: Adaptation of a visualized loop-mediated isothermal amplification technique for field detection of Plasmodium vivax infection. Parasit Vectors 2011, 4:115.

10. Mens PF, Schoone GJ, Kager PA, Schallig HDFH: Detection and identification of human Plasmodium species with real-time quantitative nucleic acid sequence-based amplification. Malar J 2006, 5:80.

11. Kosack CS, Naing WT, Piriou E, Shanks L: Routine parallel diagnosis of malaria using microscopy and the malaria rapid diagnostic test SD 05FK60: the experience of Medecins Sans Frontieres in Myanmar. Malar J 2013, 12:167.

12. Singh N, Shukla MM, Shukla MK, Mehra RK, Sharma S, Bharti PK, Singh MP, Singh A, Gunasekar A: Field and laboratory comparative evaluation of rapid malaria diagnostic tests versus traditional and molecular techniques in India. Malar J 2010, 9:191.

13. Broek IVF Van D, Van Der WS, Talukder L, Chakma S, Brockman A, Nair S, Anderson TC: Drug resistance in Plasmodium falciparum from the Chittagong hill tracts, Bangladesh. Trop Med Int Health 2004, 9:680-687.

14. Fuehrer H-P, Starzengruber P, Swoboda P, Khan WA, Matt J, Ley B, Thriemer K, Haque R, Bin YE, Hossain SM, Walochnik J, Noedl H: Indigenous Plasmodium ovale malaria in Bangladesh. Am J Trop Med Hyg 2010, 83:75-78.

15. Mohapatra PK, Prakash A, Bhattacharyya DR, Goswami BK, Ahmed A, Sarmah B, Mahanta J: Detection and molecular confirmation of a focus of Plasmodium malariae in Arunachal Pradesh, India. Indian J Med Res 2008, 128:52-56.

16. Malaria Situation in Myanmar. www.actmalaria.net/downloads/pdf/info/ 2009/Myanmar.pdf. 
17. Mens $P$, Spieker N, Omar S, Heijnen M, Schallig H, Kager PA: Is molecular biology the best alternative for diagnosis of malaria to microscopy? a comparison between microscopy, antigen detection and molecular tests in rural Kenya and urban Tanzania. Trop Med Int Health 2007, 12:238-244

18. Endeshaw T, Gebre T, Ngondi J, Graves PM, Shargie EB, Ejigsemahu Y, Ayele B, Yohannes G, Teferi T, Messele A, Zerihun M, Genet A, Mosher AW, Emerson PM, Richards FO: Evaluation of light microscopy and rapid diagnostic test for the detection of malaria under operational field conditions: a household survey in Ethiopia. Malar J 2008, 7:118.

19. Endeshaw T, Graves PM, Biru E, Gebre T, Ayele B, Yohannes G, Zerihun M, Genet A, Melak B, Kebede A, Jima D, Tadesse Z, Ngondi J, Mosher AW, Richards FO, Emerson PM: Comparison of parascreen Pan/Pf, paracheck Pf and light microscopy for detection of malaria among febrile patients, northwest Ethiopia. Trans R Soc Trop Med Hyg 2010, 104:467-474.

20. Ashton RA, Kefyalew T, Tesfaye G, Counihan H, Yadeta D, Cundill B, Reithinger R, Kolaczinski JH: Performance of three multi-species rapid diagnostic tests for diagnosis of Plasmodium falciparum and Plasmodium vivax malaria in Oromia regional state Ethiopia. Malar J 2010, 9:297.

21. Bendezu J, Rosas A, Grande T, Rodriguez H, Llanos-cuentas A, Escobedo J, Gamboa D: Field evaluation of a rapid diagnostic test (Parascreen ${ }^{T M}$ ) for malaria diagnosis in the Peruvian Amazon. Malar J 2010, 9:154.

22. Tahar R, Sayang C, Ngane Foumane V, Soula G, Moyou-Somo R, Delmont J, Basco LK: Field evaluation of rapid diagnostic tests for malaria in Yaounde, Cameroon. Acta Trop 2013, 125:214-219.

23. Endeshaw T, Graves PM, Ayele B, Mosher AW, Gebre T, Ayalew F, Genet A, Mesfin A, Shargie EB, Tadesse Z: Performance of local light microscopy and the ParaScreen Pan/Pf rapid diagnostic test to detect malaria in health centers in Northwest Ethiopia. PLoS One 2012, 7(4):e33014.

24. Nigussie D, Legesse M, Animut A, H Mariam A, Mulu A: Evaluation of Paracheck pf and Parascreen pan/pf tests for the diagnosis of malaria in an endemic area, South Ethiopia. Ethiop Med J 2008, 46:375-381.

25. World Health Organization (WHO): Basic Malaria Microscopy - Part I. Learner's Guide. 2nd edition; 2010. http://whqlibdoc.who.int/publications/2010/ 9789241547826_eng.pdf.

26. Eibach D, Traore B, Bouchrik M, Coulibaly B, Coulibaly N, Siby F, Bonnot G, Bienvenu A-L, Picot S: Evaluation of the malaria rapid diagnostic test VIKIA malaria Ag Pf/Pan ${ }^{\text {TM }}$ in endemic and non-endemic settings. Malar J 2013, 12:188.

27. WHO/FIND/CDC/TDR: Malaria rapid diagnostic test performance: results of WHO product testing of malaria RDTs: round 4. 2012, 4:22. http://apps. who.int/iris/bitstream/10665/77748/1/9789241504720_eng.pdf.

28. Abba K, Jj D, PI O, Cm N, Sm J, Takwoingi Y, Donegan S, Garner P: Rapid diagnostic tests for diagnosing uncomplicated $P$. falciparum malaria in endemic countries (Review). Cochrane Database Syst Rev 2011, 6, CD008122.

29. Lee N, Baker J, Andrews KT, Gatton ML, Bell D, Cheng Q, McCarthy J: Effect of sequence variation in Plasmodium falciparum histidine- rich protein 2 on binding of specific monoclonal antibodies: Implications for rapid diagnostic tests for malaria. J Clin Microbiol 2006, 44:2773-2778.

30. Gamboa D, Ho M-F, Bendezu J, Torres K, Chiodini PL, Barnwell JW, Incardona S, Perkins M, Bell D, McCarthy J, Cheng Q: A large proportion of $P$. falciparum isolates in the Amazon region of Peru lack pfhrp2 and pfhrp3: implications for malaria rapid diagnostic tests. PLoS One 2010, 5:e8091.

31. Kumar N, Pande V, Bhatt RM, Shah NK, Mishra N, Srivastava B, Valecha N, Anvikar AR: Genetic deletion of HRP2 and HRP3 in Indian Plasmodium falciparum population and false negative malaria rapid diagnostic test. Acta Trop 2013, 125:119-121.

32. Pava Z, Echeverry DF, Díaz G, Murillo C: Large variation in detection of histidine-rich protein 2 in Plasmodium falciparum isolates from Colombia. Am J Trop Med Hyg 2010, 83:834-837.

33. Jang JW, Cho CH, Han ET, An SS, Lim CS: pLDH level of clinically isolated Plasmodium vivax and detection limit of pLDH based malaria rapid diagnostic test. Malar J 2013, 12:181.

doi:10.1186/1475-2875-12-378

Cite this article as: Elahi et al:: Performance of a HRP-2/pLDH based rapid diagnostic test at the Bangladesh-India-Myanmar border areas for diagnosis of clinical malaria. Malaria Journal 2013 12:378.

\section{Submit your next manuscript to BioMed Central and take full advantage of:}

- Convenient online submission

- Thorough peer review

- No space constraints or color figure charges

- Immediate publication on acceptance

- Inclusion in PubMed, CAS, Scopus and Google Scholar

- Research which is freely available for redistribution 